\title{
ORGANISMS ISOLATED IN INDUCED SPUTUM SAMPLES IN ACUTE EXACERBATION OF SEVERE AND VERY SEVERE CHRONIC OBSTRUCTIVE PULMONARY DISEASE

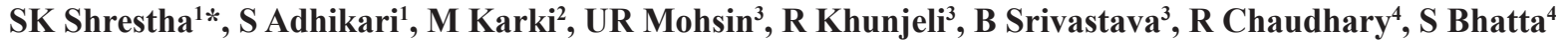

\author{
${ }^{1}$ Department of Pulmonary, Critical Care and Sleep Medicine, NAMS, Kathmandu \\ ${ }^{2}$ Oxford university Clinical Research Unit (Nepal), Patan Hospital, Lalitpur \\ ${ }^{3}$ Department of Respiratory Medicine, Shree Birendra Hospital, Kathmandu \\ ${ }^{4}$ Department of Pathology, Microbiology Unit, Shree Birendra Hospital. Kathmandu
}

*Correspondence to: Dr. Sanjeet Krishna Shrestha, DM fellow Pulmonary, Critical Care and Sleep Medicine, NAMS, Kathmandu

E-mail: shrestha.sanjeet@gmail.com

\begin{abstract}
Acute exacerbations pose a very high economic burden on Chronic Obstructive Pulmonary Disease (COPD) patients and are commonly infective in nature. Gram's staining and bacterial cultures are baseline investigations for sputum examination for COPD. This study evaluated these techniques for characterization and identification of various organism involved in acute infective exacerbations of COPD. Sodium Chloride (3\%) induced sputum samples from 122 severe and very severe COPD subjects presenting in acute exacerbation who had history of frequent exacerbations and frequent antibiotics use were evaluated. The sputum samples were evaluated by Gram's staining and bacterial culture from January 2013 to March 2014. Induction technique was able to obtain adequate samples from 86 ( $70.48 \%$ of 122$)$ subjects. Gram's stain showed 30 samples of Gram positive cocci (34.88\%), 23 samples (26.74\%) of Gram Negative Cocci and 50 samples (58.13\%) of Gram Negative Bacilli. Bacterial culture showed predominant growth of Gram negative organisms including Pseudomonas sp, Acinetobacter sp, Klebsiella pneumoniae and Citrobacter Freundii. Gram negative bacilli are the most common isolated pathogens responsible for the acute exacerbation in severe and very severe COPDs with history of frequent exacerbations and hospital visits.
\end{abstract}

Key words: Chronic obstructive pulmonary disease, Gram Negative Bacterial infections, Pseudomonas aeruginosa.

\section{INTRODUCTION}

Chronic Obstructive Pulmonary Disease (COPD) is an ongoing epidemic which is increasing in incidence and is life threatening. It was responsible for three million deaths in 2012 which amounted to $6 \%$ of global deaths. ${ }^{1,2}$ Prevalence of COPD varies with region and survey method ranging from 5\% to $19.7 \%$ with most regions showing average range between 5 to $10 \% .^{1,2,3}$

Major cause for morbidity and mortality in COPD is exacerbations with all-cause mortality reaching as high as $49 \%$ after three years of hospital admission. ${ }^{3}$ Bacterial and viral infection of respiratory track is a major cause for exacerbations. ${ }^{3}$ The colonization of pathogenic bacteria in lower respiratory tract is common in $\mathrm{COPD}^{4,5}$ with increase in bacterial growth during acute exacerbation. ${ }^{5}$ The bacterial cultures largely rely on expectorated sputum evaluation which usually reveal Gram positive Cocci (GPCs) 
Shrestha et al, Journal of Chitwan Medical College 2015; 5(13)

and Gram Negative Bacilli (GNBs). The GNBs like

Pseudomonas, Klebsiella and Serratia predominates with deterioration of lung function in COPD. ${ }^{6}$

We evaluated the induced sputum samples by Gram's staining and conventional bacterial cultures to characterize the organisms involved in acute exacerbations (AE) in severe and very severe COPD patients with history of frequent exacerbations and hospital visits.

\section{MATERIALS AND METHODS}

\section{Study population}

The study included 122 severe and very severe COPD patients who were on regular standard optimal medical therapy. The patients presenting to department of emergency with acute exacerbation were enrolled. The patients previously diagnosed of COPD by spirometry and already on treatment were selected. Only those patients with frequent COPD exacerbations (more than two episodes in a year) and frequent hospital visits were included. The patients were enrolled in the study from January 2013 to March 2014 (16 months). The clinical definition of AE of COPD was as per standard guidelines of Global initiative for Obstructive Lung Disease (GOLD) ${ }^{3}$ which included change in day to day symptoms of breathlessness, cough, increased sputum purulence and amount. Other respiratory diseases were excluded clinically and by chest X-ray.
Sample collection

Analysis was done in all the 122 subjects as they presented in the hospital. Two sets of sputum samples were collected from each subject on the first day. All samples were collected after rinsing the mouth with sterile water. Sputum samples were induced using 3\% Sodium Chloride $(\mathrm{NaCl})$. First, patients were given nebulization via nebulizer with Salbutamol. Then patients were started on $3 \% \mathrm{NaCl}(5 \mathrm{ml})$ nebulization for 15 to 20 minutes. The patients were asked to expectorate every five minutes or whenever they feel like being able to expectorate during the time frame. If a patient was not able to expectorate within 20 minutes of the procedure, procedure was abandoned. These samples were immediately submitted to the microbiology lab and processed within two hours. Induced sputum samples were taken up for Gram's staining and culture in a sterile sputum specimen container. $^{7}$

\section{Sample processing}

The sputum samples were subjected to macroscopic evaluation. Sputum and saliva were separated mechanically. The prepared samples were first subjected to Gram's staining from the area of maximum purulence. The number of polymorphonuclear (PMN) leukocytes and squamous epithelial cells were identified. The sample having less than 10 squamous epithelial cells and more than 25 PMN Leukocytes were labelled as adequate. All 
samples were subjected to conventional bacterial microscopic verification and processing.

culture. Gram positive organisms were cultured in blood agar. Gram negative organisms were cultured in MacConkey agar and enriched chocolate agar. The plates were incubated at $37^{\circ} \mathrm{C}$ and $5 \%$ Carbon dioxide. Microbiological standards were used for identification of organisms.

\section{STATISTICAL ANALYSIS}

The initial data entry was done using Microsoft Excel 2010 and further statistical analysis was done with IBM SPSS version 20. All the categorical data were expressed in percentage and absolute numbers and continuous numerical data were expressed as mean \pm Standard deviation. The statistical significance was set at $\mathrm{p}<0.05$ with $95 \%$ confidence. The statistical evaluation for categorical variables were done using chi square test.

\section{RESULTS}

Demographic profile of the patients at baseline

A total of 122 subjects with frequent AE of COPD were included prospectively in the study. The mean age of the study population was $66.11 \pm 10.75$ years. There were total of 63 females (51.6\%) and 59 males (48.4\%) with the male: female ratio of 1.06:1. Sputum samples were subjected to Gram's staining and routine bacterial cultures. All 122 subjects produced adequate sputum samples on gross inspection at the bedsides. These samples were immediately submitted to the microbiology laboratory for further

\section{Gram's Stain}

Among the 122 samples collected and submitted for Gram's staining 36 samples (29.50\%) were identified to be not representative of lower respiratory tract as per microscopic definition. The remaining 86 adequate samples $(70.48 \%)$ were evaluated for presence of various Organisms. The processed samples revealed bacteria in 73 samples $(84.88 \%$ of 86$)$ whereas 13 samples $(15.11 \%$ of 86$)$ were negative. A total of 30 samples (34.88\% of 86$)$ showed GPCs. Only one sample showed $(1.16 \%$ of 86$)$ Gram positive bacilli (GPB). Twenty three samples $(26.74 \%$ of 86) showed Gram Negative Cocci (GNC) and 50 samples (58.13\% of 86) showed GNBs. GPCs and GNBs were seen mixed in 11 samples $(12.79 \%$ of 86). GPCs and GNCs were seen in mixed population in 13 samples (15.11\% of 86). GNCs and GNBs were seen together in five samples (5.81\% of 86$)$.

\section{Conventional Bacterial Cultures}

All 122 samples of sputum collected in sterile container for culture were subjected to conventional bacterial cultures. Among these samples 31 samples $(25.41 \%$ of 122) were representative of upper respiratory tract organisms and hence non-pathogenic. Thirteen samples $(10.66 \%$ of 122$)$ did not show any growth on bacterial culture media. Only 78 sputum samples $(63.93 \%$ of 122$)$ showed growth of organism of significance and hence considered pathogenic. 
Among the 78 cultures showing significant growth predominant isolates were of Gram negative organisms which accounted for total 72 cultures $(92.31 \%$ of 78$)$. There were only six cultures (7.69\% of 78 ) showing growth of Gram positive organisms. Among the isolated GNBs, Pseudomonas aeruginosa was isolated in 24 samples $(30.77 \%$ of 78). Acinetobacter sp. were isolated in 16 sputum samples (20.51\% of 78$),$ Klebsiella sp. were isolated in 15 sputum samples $(19.23 \%$ of 78$)$. There were 13 isolates (16.67\% of 78 ) of Klebsiella pneumoniae and 2 isolates $(2.56 \%$ of 78$)$ of Klebsiella oxytoca. There were nine samples (11.54\%) showing growth of Escherichia coli. There were five samples (6.41\% of 78) showing growth of Citrobacter sp. This included four Citrobacter freundii isolates $(5.13 \%$ of 78 ) and one Citrobacter koseri (1.28\% of 78$)$. There were two samples $(2.56 \%$ of 78$)$ showing growth of Moraxella catarrhalis and one sample (1.28\% of 78$)$ showing growth of Hafnia sp.
Table 1 Organisms isolated in the sputum samples of patients with acute exacerbation of COPD (percentage expressed with respect to 122 samples)

\begin{tabular}{|c|c|c|}
\hline Organisms & $\begin{array}{l}\text { Number/ } \\
\text { Frequency }\end{array}$ & Percent \\
\hline \multicolumn{3}{|c|}{ Clinically insignificant Growths (36.07\%) } \\
\hline No Growth & 13 & 10.66 \\
\hline URT organisms & 31 & 25.41 \\
\hline \multicolumn{3}{|c|}{ Clinically significant Growths (63.93) } \\
\hline $\begin{array}{l}\text { Gram Negative Organ- } \\
\text { isms }\end{array}$ & 72 & $59.01 \%$ \\
\hline Pseudomonas aeruginosa & 24 & 19.67 \\
\hline Acinetobacter sp. & 16 & 13.11 \\
\hline Klebsiella pneumonia & 13 & 10.65 \\
\hline Klebsiella oxytoca & 2 & 1.64 \\
\hline Escherichia coli & 9 & 7.38 \\
\hline Citrobacter freundii & 4 & 3.28 \\
\hline Citrobacter koseri & 1 & 0.82 \\
\hline Moraxella catarrhalis & 2 & 1.64 \\
\hline Hafnia sp. & 1 & 0.82 \\
\hline $\begin{array}{l}\text { Gram Positive Organ- } \\
\text { isms }\end{array}$ & 6 & $4.92 \%$ \\
\hline Streptococcus sp. & 2 & 1.64 \\
\hline Staphylococcus & 2 & 1.64 \\
\hline MRSA & 1 & \\
\hline CoNS & 1 & \\
\hline Enterococcus & 2 & 1.64 \\
\hline
\end{tabular}

A total of six samples showed growth of GPCs. This included Streptococcus sp. in two sputum samples (2.56\% of 78). Staphylococcus was isolated in two sputum samples $(2.56 \%$ of 78$)$ including one Methicillin Resistant Staphylococcus Aureus (MRSA) and one culture of Coagulase negative SA (CoNS). Enterococcus sp. was isolated in two samples $(2.56 \%)$. 


\section{DISCUSSION}

Shrestha et al, Journal of Chitwan Medical College 2015; 5(13)

Acute infective exacerbation of COPD is the most common cause for morbidity in COPD. Both viral and bacterial aetiologies are implicated. ${ }^{3}$ Bacterial growths of clinical significance and pathological potential can be identified it majority of $\mathrm{AE}$ COPD patients. It can range from $50 \%$ to $80 \%$. It is observed that almost $25 \%$ of chronic stable COPDs harbour potential bacterial pathogens. ${ }^{9}$ Studies undergoing bronchoscopic evaluation of AE COPD show Haemophilus influenzae, Moraxella catarrhalis, Streptococcus pneumoniae and Pseudomonas aeruginosa as the most commonly isolated organisms. ${ }^{8}$ It is seen that the microbiome and their involvement in acute exacerbation also depends on various factors including the deterioration of forced expiratory volume in first second (FEV1). ${ }^{6}$ As the FEV1 starts decreasing there is increasing involvement of GNBs in the pathogenesis of $\mathrm{AE}$ COPD. These GNBs usually are Enterobacteriaceae group: Pseudomonas sp, Serratia sp, Klebsiella sp, Citrobacter sp, Proteus sp, E. coli and Enterobacter $\mathrm{sp}^{6}$

Our study involved severe $(\mathrm{FEV} 1<50 \%)$ and very severe (FEV1<30\%) COPD subjects with history of frequent exacerbations and frequent use of antibiotic during hospital visits. Similar pattern of involvement of organisms were observed in severe and very severe COPD. We observed that predominant isolates were GNBs (92.31\%), which was in accordance to research done by Eller $\mathrm{J}$ et al. ${ }^{6}$ Pseudomonas aeruginosa was the most common organism isolated $(30.77 \%)$ in our study. Study conducted by Gallego M. et al. ${ }^{10}$ have shown involvement of Pseudomonas sp. in infectious AE COPD to be around 34.7\%. Severe forms of COPD, reduced FEV1, frequent use of antibiotics and recent hospitalizations are identified as predispositions for Pseudomonas infection in COPD. ${ }^{6,11,12}$ The patient demographics in our study matched the afore mentioned characteristics hence higher number of isolates were Pseudomonas. Remaining of Enterobacteriaceae group of organisms are also isolated with increasing frequency with sequential decline of lung functions. ${ }^{6}$ Our study also showed considerable growths of Klebsiella pneumoniae, E. coli and Citrobacter freundii. There are studies which show significant higher growths of Enterobacteriaceae in severe $\mathrm{COPD}^{6,10}$ On the contrary, it is identified that conventional bacterial culture methods tend to overestimate Enterobacteriaceae group when compared with newer pyrosequencing methods in COPD. ${ }^{13}$ Aguirre et al. in the same study also identified under-estimation of difficult to grow organisms by conventional techniques. ${ }^{13}$ This limitation is also applicable to our study which utilized conventional methods for bacteriological culture, yielding higher 
GNBs as compared to GPCs and also poor yield of Haemophilus influenza. In addition we utilized induced sputum sample but invasive studies like bronchoscopy were not done.

Induced sputum sample is a reliable, safe and comparable method of sampling in AE COPD patients for obtaining sputum samples. ${ }^{15}$ In our study it was able to produce adequate specimen of sputum in $70.48 \%$ of the samples. This is comparable with studies done on efficacy of sputum samples with value of $63.1 \%$ in Tuberculosis patients. ${ }^{15}$ The procedural efficacy was $86 \%$ in AE COPD patients in the study done by Zeng M. et al. ${ }^{14}$

\section{CONCLUSION}

Induced sputum samples can produce adequate samples for both Gram's staining and Bacterial culture. Gram negative organisms are predominantly involved in infective exacerbation of severe and very severe COPDs after frequent exacerbations and frequent use of antibiotics. Pseudomonas aeruginosa, Acinetobacter sp, Klebsiella pneumoniae and Escherichia coli are the commonest Gram negative pathogens involved.

\section{REFERENCES}

1. Salvi S. Chronic Obstructive Pulmonary Disease: The neglected epidemic. In: Jindal Sk, Shankar PS, Raoof S, Gupta D, Aggarwal AN, Agarwal R (eds) Textbook of Pulmonary and Critical Care Medicine. New Delhi, Jaypee Brothers Medical
Publishers; 2011. p 971.

2. Who.int. World Health Organisation. Chronic obstructive pulmonary disease (COPD) [Internet]. 2015 [cited 2 June 2015].

3. Global Strategy for the Diagnosis, Management and Prevention of COPD, Global Initiative for Chronic Obstructive Lung Disease (GOLD) [Internet]. 2015 [cited 2 June 2015].

4. Sethi S, Murphy TF. Infection in pathogenesis and course of chronic obstructive pulmonary disease. N Engl J Med 2008;359:2355-65.

5. Pela R, Marchesani F, Agostinelli C, Staccioli D, Cecarini L, Bassotti C, Sanguinetti CM. Airways microbial flora in COPD patients in stable clinical conditions and during exacerbations: a bronchoscopic investigation. Monaldi Arch Chest Dis. 1998 Jun;53(3):262-7.

6. Eller J, Ede A, Schaberg T, Niederman MS, Mauch H, Lode H. Infective exacerbations of chronic bronchitis: relation between bacteriologic etiology and lung function. Chest. 1998 Jun;113(6):1542-8.

7. Gupta KB, Garg S. Sputum induction- A useful tool in respiratory diseases. Lung India 2006;23:82-86.

8. Iyer Parameswaran G, Murphy TF. Chronic obstructive pulmonary disease: role of bacteria and updated guide to antibacterial selection in the older patient. Drugs Aging. 2009;26(12):985-95. 
9. Rosell A, Monsó E, Soler N, Torres F, Angrill $\mathrm{J}$, Riise $\mathrm{G}$, et al. Microbiologic determinants of exacerbation in chronic obstructive pulmonary disease. Arch Intern Med. 2005 Apr $25 ; 165(8): 891-7$.

10. Gallego M, Pomares X, Espasa M, Castañer E, Solé M, Suárez D, et al.Pseudomonas aeruginosa isolates in severe chronic obstructive pulmonary disease: characterization and risk factors. BMC Pulm Med. 2014 Jun 26;14:103.

11. Liu S, Zhou Y, Wang X, et al. Biomass fuels are the probable risk factor for chronic obstructive pulmonary disease in rural South China. Thorax 2007 Oct; 62 (10): 889-97.

12. Sullivan SD, Ramsey SD, Lee TA. The economic burden of COPD. Chest 2000 Feb; 117 (2 Suppl.): $5 S-9 S$.
13. Aguirre E, Galiana A, Mira A, Guardiola R, Sánchez-Guillén L, Garcia-Pachon E. et al. Analysis of microbiota in stable patients with chronic obstructive pulmonary disease. APMIS. 2015 May;123(5):427-32.

14. Zeng M, Wu JF, Xie CM, Yan YS, Chen DM, Xuan JM. Safety of sputum induction in subjects with acute exacerbations of chronic obstructive pulmonary disease. Zhonghua Jie $\mathrm{He} \mathrm{He} \mathrm{Hu} \mathrm{Xi}$ Za Zhi. 2005 Apr;28(4):238-41.

15. Seong GM, Lee J, Lee JH, Kim JH, Kim M. Usefulness of sputum induction with hypertonic saline in a real clinical practice for bacteriological yields of active pulmonary tuberculosis. Tuberc Respir Dis (Seoul). 2014 Apr;76(4):163-8. 